CASE REPORTS / REPORTE DE CASO

\title{
Myopericarditis and skin rash in a patient with COVID-19 infection
}

Araque R Jorge ${ }^{1}$, Paez C Jhenina ${ }^{2}$

DOI. 10.21931/RB/2020.05.03.16

Abstract: In the current COVID-19 pandemic, it should be the first diagnosis to be excluded, even in endemic areas of other diseases. The COVID-19 disease can be presented with cutaneous manifestations, especially in children. Cardiovascular complications must be taken into account since they can be undiagnosed in patients who do not develop symptoms.

Key words: COVID 19, myopericarditis, skin rash.

\section{Introduction}

Girl from an area endemic to tropical diseases, with fever and a diffuse polymorphic rash, is diagnosed with COVID 19. She develops myopericarditis with good clinical evolution.

A 1-year-10-month-old girl, from Manta-Ecuador (tropical climate region), with no history of cardiovascular disease, arrived, accompanied by her parents, to the emergency department of a private medical center in the city of Quito, for presenting persistent fever of $38^{\circ} \mathrm{C}$ for 15 days. The clinical picture evolved on the second day with bilateral conjunctivitis (Figure 2) without discharge, a generalized polymorphic rash that resolved spontaneously in 8 days (Figure $1 \mathrm{~A}, \mathrm{~B}, \mathrm{C}$ ) with subsequent light peeling of the skin; edema in eyelids, hands, and feet, general discomfort, occasional cough, and light arthralgias. On physical examination: $T^{\circ}$ : $38.6 \mathrm{C}^{\circ}, \mathrm{HR}: 120$ bpm, RR: 26 x', SatO2: 92\% ambient air. Heart: heart sounds, rhythms, no murmurs. Pulmonary: preserved vesicular murmur.

\section{Clinical case}

\section{Differential diagnosis}

The differential diagnosis includes childhood virosis, Kawasaki disease, Dengue, chikungunya, COVID-19 infection, a bacterial infection.

\section{Investigation}

In laboratory data (Table 1), the blood cell count revealed leukocytosis, neutrophilia, lymphopenia, normochromic normocytic anemia, thrombocytosis, healthy kidney and liver function, elevated C-reactive protein, normal procalcitonin. A study was carried out for different viral agents according to the clinical and epidemiological conditions, which were negative (Table 1). Three blood cultures performed did not report bacterial growth. Chest X-ray reported accentuation of the bilateral perihilar axial peribronchovascular interstitium, dis-
A

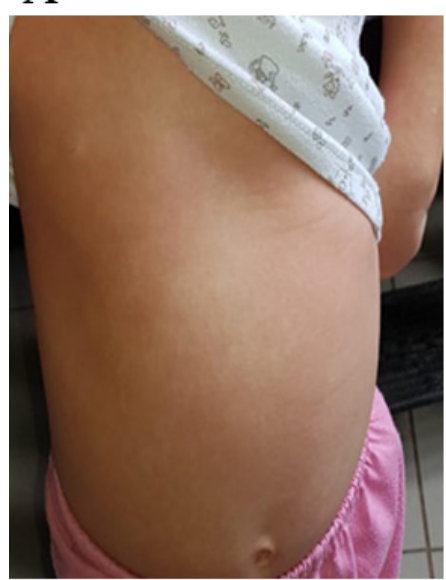

$\mathrm{B}$

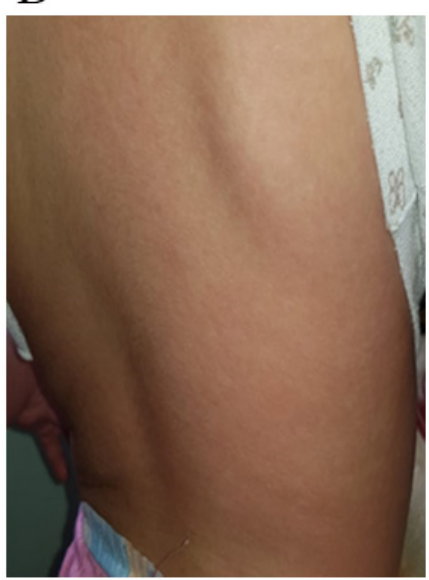

$\mathrm{C}$

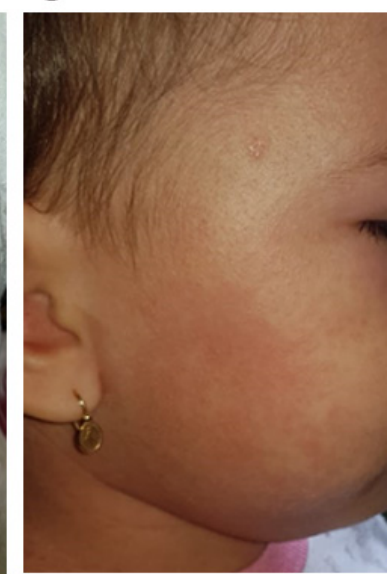

Figure 1. Generalized polymorph exanthem. A: Anterior thorax and upper limb; B: Posterior thorax; C: face.

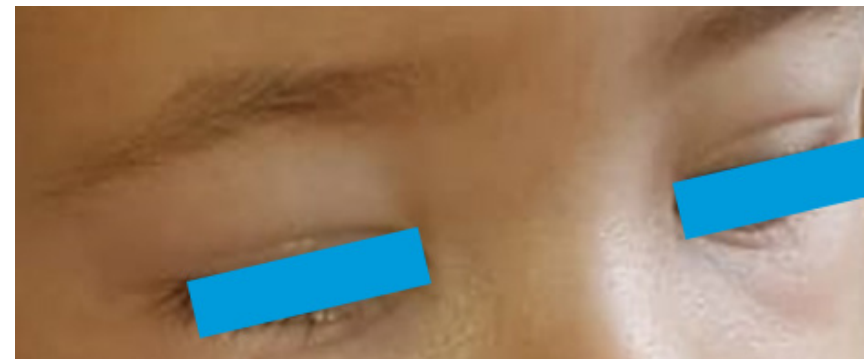

Figure 2. Eyelid edema and bilateral conjunctivitis

${ }^{1}$ Cardiologist, International Clinic, Quito, Ecuador.
${ }^{2}$ Radiologist, International Clinic, Quito, Ecuador.

Corresponding author: jordi_var@yahoo es 
crete right paracardiac acinar infiltrante, and discrete increase in the size of the cardiac silhouette (Figure 3). In the echocardiogram (figure 4A), we observed cardiac chambers of size within normal limits for age, a standard diameter of coronary arteries. Light circumferential pericardial effusion, with $2 \mathrm{~mm}$ of separation between pericardial blades, more evident in right chambers without signs of cardiac tamponade. The pulmonary echo reported slight irregularities of the pleural line in zone 10 (Figure 6A). Analyses were complemented by a laboratory study, observing a nasopharyngeal swab a positive for SARSCoV-2 in the real-time reverse transcriptase-polymerase chain reaction, and elevation of ferritin (329), lactic dehydrogenase (546 U / L) and troponin T (29.10 pg / mL).

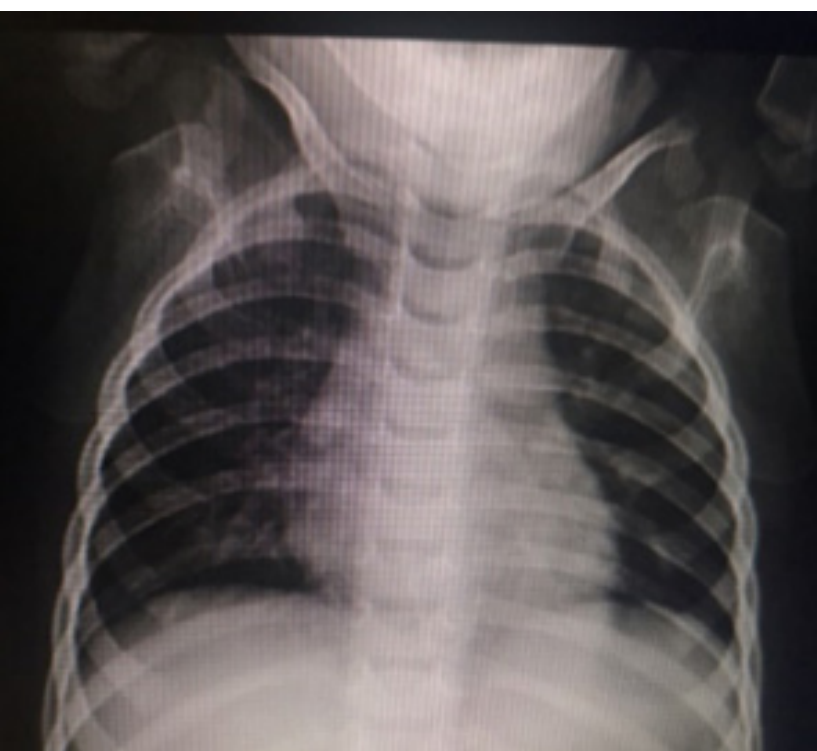

Figure 3. Chest A-P x-ray: accentuation of the bilateral para-hilar axial peribronchovascular interstitium, discrete right paracardiac acinar infiltrante, and discrete increase in the size of the right cardiac silhouette.

\section{Treatment}

During her hospitalization, the patient maintained hemodynamic stability. Treatment with antibiotics (azithromycin, ampicillin/sulbactam), paracetamol, and intravenous hydration was started, observing clinical and laboratory improvement. In the ultrasound control carried out 72 hours later, she reported areas of pericardial thickening $(4 \mathrm{~mm})$, hyper-echogenic along the left ventricular wall, and minor effusion without signs of tamponade (Figure 4B), for this reason, it was decided to continue observing. The pulmonary echo showed normalization of the pleural line in segment 10 (Figure 5B). The patient was discharged on day 11 . Telephone follow-up was performed 15 days later; the parents stated that the girl was still asymptomatic.

\section{Discussion}

Coronaviruses (CoV) are a family of viruses that can cause various diseases, from a common cold to more severe diseases, such as the Middle East respiratory syndrome (MERS$\mathrm{CoV}$ ) and severe acute respiratory syndrome (SARS-CoV). The current disease outbreak is caused by a new strain of coronavirus called CoV-19 first reported in Wuhan (China) on 31 December 2019. Ecuador reported its first case on 29 February 2020

The first epidemiological data on children reported in $\mathrm{Wu}$ han, China, indicate that of 366 children hospitalized for respiratory conditions between January 7 and 15, 2020, 1.6\% were positive for COVID 19?

The incubation period of the virus is from 2 to 12 days, with an average of 5.1 days. The clinical picture of SARS-CoV-2 infection is characterized primarily by symptoms of the respiratory tract, including fever (in up to $88.7 \%$ of hospitalized patients), cough (67.8\% of patients), pharyngodynia, fatigue, conjunctivitis and complications related to pneumonia and acute respiratory distress syndrome ${ }^{1}$. As the disease has spread throughout the world, several cases of extrapulmonary invol-

\begin{tabular}{|c|c|c|c|c|}
\hline \multirow[t]{2}{*}{ Parameter } & \multicolumn{4}{|c|}{ Result } \\
\hline & Reference & Day 1 & Day 4 & Day 11 \\
\hline White dlood cell count,per K/uL & $4.80-10.80$ & 25.59 & 20.64 & 14.81 \\
\hline \multicolumn{5}{|l|}{ Lymphocyte count } \\
\hline Relative, $\%$ & $30.0-42.0$ & 16.9 & 19.6 & 36.9 \\
\hline Absoluto, K/ uL & $1.00-7.00$ & 4.33 & 4.04 & 5.47 \\
\hline Platelet count, $\mathrm{K} / \mathrm{uL}$ & $130.000-400.00$ & 639.000 & 608.000 & 908.00 \\
\hline Red blood cell, M/uL & $3.80-5.30$ & 3,91 & 3.53 & 3.71 \\
\hline Hemoglobin g/dl & $10.5-14.4$ & 10.1 & 9.2 & 9.2 \\
\hline Hematocrit,\% & $32.0-43.0$ & 30.1 & 27.1 & 28.2 \\
\hline Creatinine , mg/dL & $0.50-0.90$ & 0.5 & 0.5 & 0.5 \\
\hline C-reactive protein, $\mathrm{mg} / \mathrm{L}$ & $0.10-2.80$ & 313.08 & 224.75 & 154 \\
\hline Procalcitonine, $\mathrm{mg} / \mathrm{mL}$ & $<0.46$ & 0.21 & 0.18 & 0.13 \\
\hline Ferritin & $10.9-92.2$ & N/A & 329 & N/A \\
\hline Lactate dehydrogenase, U/L & $225.00-450-00$ & 546.00 & N/A & N/A \\
\hline Sodium, $\mathrm{mEq} / \mathrm{L}$ & $135-155$ & 135 & N/A & N/A \\
\hline Potassium, $\mathrm{mEq} / \mathrm{L}$ & $3.50-5.50$ & 5.30 & N/A & N/A \\
\hline Choride, $\mathrm{mEq} / \mathrm{L}$ & $95-116$ & 100 & N/A & N/A \\
\hline Creatine Kinase-MB, ng/mL & $0.00-25.00$ & N/A & 18.10 & N/A \\
\hline troponinT, $\mathrm{pg} / \mathrm{mL}$ & $<14$ & N/A & 29.10 & N/A \\
\hline Influenza A/B & & negative & N/A & N/A \\
\hline Adeno virus respiratorio & & Negative & N/A & N/A \\
\hline Virus sincitial respiratory & & negative & N/A & N/A \\
\hline Citomegalovirus IgG , U/ml & $<0.5$ & $<0.150$ & N/A & N/A \\
\hline
\end{tabular}

Table 1. Laboratory clinical result. 

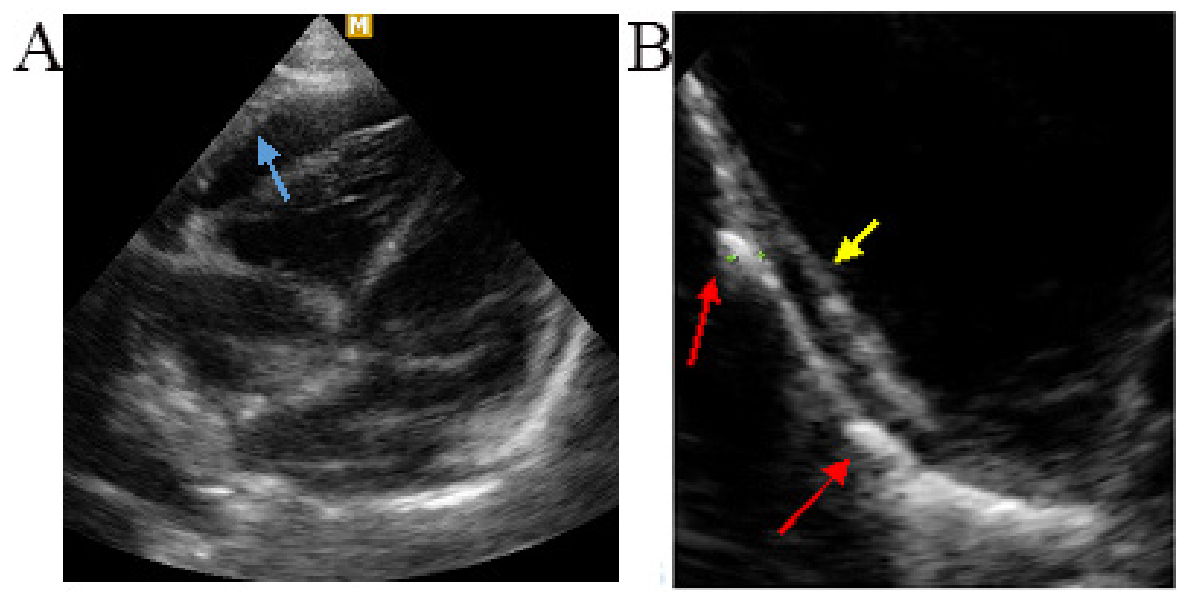

Figure 4. Apical 4-chamber echocardiogram, showing the most significant pericardial effusion at the level of the right cavities (light blue arrow). B: zoom axis for longitudinal sternal, more refractive parietal pericardial thickening areas (red arrows). Visceral pericardium (yellow arrow).
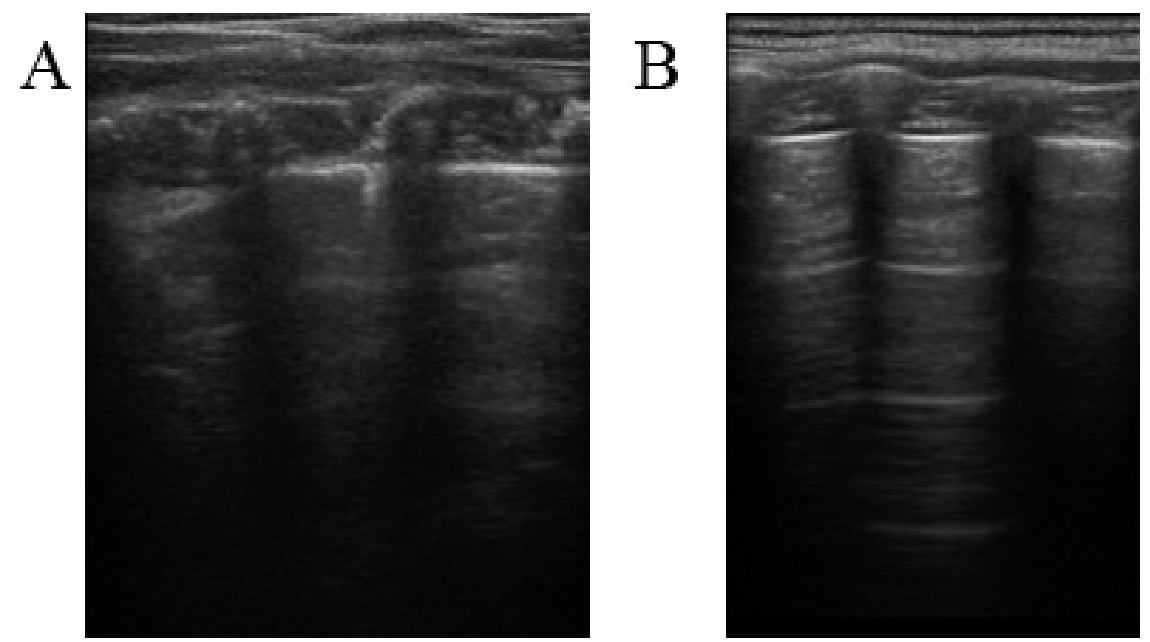

Figure 5. pulmonary echo may show slight irregularity of the pleural line with inconspicuous A-lines; B: study performed 5 days later in which normalization of the pleural line was observed with visualization of lines A.

vement have been reported (e.g., gastrointestinal, skin, cardiovascular, nervous system). From what has been reported so far, the skin manifestations related to COVID-19 may appear at the beginning, during or at the end of the disease, they are very varied and nonspecific and would resolve spontaneously. The most frequent lesions are erythematous rash, generalized urticaria, varicelliform rash, and acro-ischemic lesions-all of them with little itching and unrelated to the severity of the infection. Treatment of urticarial rash, rash, or vesicular eruption is symptomatic (topical antihistamines, emollients, antiseptics, and corticosteroids), and in more complex cases such as acro-ischemic lesions, low molecular weight heparins are indicated ${ }^{3,4,5}$. In our case, the rash appeared at the beginning of the clinical picture, with spontaneous resolution after 8 days and subsequent light peeling of the skin.

Differential diagnosis is complicated if we take into account that several childhood viral infections are presented with skin lesions, conjunctivitis, and fever. If we also add the fact that endemic diseases that trigger similar symptoms coexist in the patient's geographic area of residence (Dengue, chikungunya, and zika), we can understand that differential diagnosis is a real challenge for the medical community to face in the current COVID-19 pandemic.

The relationship between COVID 19 infection and pericardial manifestations are rare. The COVID 19 virus is likely to trigger an inflammatory response leading to pericarditis and, subsequently, pericardial effusion. Elevation of cardiac troponin levels is a sensitive marker of myocardial injury associated with pericarditis, as in the present case, and is called myopericarditis. Driggin et al. ${ }^{6}$ reported that increased troponin levels occur in $7 \%$ of patients who developed fulminant myocarditis.

The exact mechanism of affectation is unclear, but it is presumed that the virus could cause inflammation of the pericardium and myocardium by a direct cytotoxic effect and by immune-mediated mechanisms ${ }^{7}$. Myopericarditis associated with other cardiotropic viruses, such as influenza and parvovirus B-19, have been extensively described ${ }^{7}$. However, little is known about cardiac involvement as a complication of $\mathrm{CO}$ VID-19 infection. Our patient developed a subclinical picture of myopericarditis, maintaining the optimal bi-ventricular function, without showing signs of cardiac tamponade and with excellent clinical and ultrasound evolution. Cardiovascular complications are probably mild and well-tolerated in childre

The sensitivity and specificity of the imaging methods in the diagnosis of COVID-19 infection are variable; the computed tomography scan has a low sensitivity (around 50\%) in those patients who have constitutional symptoms and do not have respiratory symptoms, and high in patients with a positive study for COVID 19 (86 to 97\% in different studies). The chest X-ray has a sensitivity of 59\%. Ultrasound studies have 
a very low specificity with an excellent sensitivity of $75 \%$ and are currently very useful in monitoring lung lesions ${ }^{1}$. The first lung echo study performed in our patient showed thickening and irregularities of the pleural line with normalization in subsequent studies, signs that can be identified in patients with a positive COVID 19.

\section{Conclusions}

The current pandemic caused by COVID 19 has changed lifestyle and has marked a substantial public health problem. It has exposed the fragile health systems in underdeveloped countries that have tried to make up numbers of contagion and deaths.

Faced with this grim picture, the fact that the clinical patterns of COVID 19 remain unclear, especially in children, is added. In tropical and subtropical regions, the presence of diseases that cause fever and skin lesions such as Dengue, chikungunya, and zika is frequent, in addition to the typical virosis of childhood. However, given the wave of COVID 19 infections, this should be the first option to rule out since there may be many patients with skin manifestations that are passing or have passed an infection with minimal symptoms and, therefore, they are not have done a coronavirus PCR. The involvement of the pericardium and the myocardium must also be taken into account in patients with COVID-19 disease, to search for it, diagnose it, and monitor its evolution to avoid complications.

\section{Bibliographic references}

1. Giuseppe Pascarella, Alessandro Strumia, Chiara Piliego, Federica Bruno, Romualdo Del Buono, Fabio Costa, Simone Scarlata \& Felice Eugenio Agrò. COVID-19 diagnosis and management: a comprehensive review. doi: 10.1111/JOIM.13091.

2. Detection of COVID-19 in Children in Early January 2020 in Wuhan, China. N Engl J Med 382;14 nejm.org 2 April, 2020.

3. Chen $Y$, Peng $H$, Wang L, Zhao $Y$, Zeng L, Gao H, et al. Infants Born to Mothers with a New Coronavirus (COVID-19). [Internet]. Front Pediatr. 2020;8. Disponible en: https://www.frontiersin.org/articles/10.3389/fped.2020.00104/full

4. Joob B, Wiwanitkit V. COVID-19 can present with a rash and be mistaken for Dengue. J Am Acad Dermatol. marzo de 2020;82(5):e177. DOI: 10.1016/j.jaad.2020.03.036

5. Zhang J, Dong X, Cao Y, Yuan Y, Yang Y, Yan Y, et al. Clinical characteristics of 140 patients infected with SARS-CoV-2 in Wuhan, China. [Internet.] Allergy. 2020. Disponible en: https://onlinelibrary. wiley.com/doi/abs/10.1111/all.14238.

6. Driggin E, Madhavan MV, Bikdeli B, et al. Cardiovascular Considerations for Patients, Health Care Workers, and Health Systems During the Coronavirus Disease 2019 (COVID-19) Pandemic. J Am Coll Cardiol 2020.

7. Riccardo M.Inciardi, MD; Laura Lupi, MD; Gregorio Zaccone, MD; Leonardoltalia, MD; Michela Raffo, MD; Daniela Tomasoni ,MD; Dario S. Cani, MD; Manuel Cerini, MD; Davide Farina, MD; Emanuele Gavazzi, MD; Roberto Maroldi, MD; Marianna Adamo, MD; Enrico Ammirati, MD, PhD; Gianfranco Sinagra, MD; Carlo M. Lombardi, MD; Marco Metra, MD. Cardiac Involvementina Patient With Coronavirus Disease 2019 (COVID-19). JAMA Cardiol. doi: 10.1001 / jamacardio.2020.1096

Received: 1 July 2020

Accepted: 10 Agost 2020 